\title{
Advances in basic science methodologies for clinical diagnosis in female stress urinary incontinence
}

\author{
Marwa Abdulaziz, $M S c^{1}$; Emily G Deegan, RN2; Alex Kavanagh, $M D^{3}$; Lynn Stothers, $M D^{3}$; Denise Pugash, $M D^{4}$; \\ Andrew Macnab, MD (London) ${ }^{3}$
}

'PhD Candidate, Department of Experimental Medicine; ${ }^{2}$ Masters Candidate, Department of Experimental Medicine; ${ }^{3}$ Department of Urologic Sciences; ${ }^{4}$ Department of Radiology, BC Women's Hospital; University of British Columbia, Vancouver, BC, Canada

Cite as: Can Urol Assoc J 2017;11 (6Suppl2):S117-20 http://dx.doi.org/10.5489/cuaj.4583

\section{Abstract}

We provide an overview of advanced imaging techniques currently being explored to gain greater understanding of the complexity of stress urinary incontinence (SUI) through better definition of structural anatomic data. Two methods of imaging and analysis are detailed for SUI with or without prolapse: 1) open magnetic resonance imaging (MRI) with or without the use of reference lines; and 2) 3D reconstruction of the pelvis using MRI. An additional innovative method of assessment includes the use of near infrared spectroscopy (NIRS), which uses non-invasive photonics in a vaginal speculum to objectively evaluate pelvic floor muscle (PFM) function as it relates to SUI pathology. Advantages and disadvantages of these techniques are described. The recent innovation of open-configuration magnetic resonance imaging (MRO) allows images to be captured in sitting and standing positions, which better simulates states that correlate with urinary leakage and can be further enhanced with 3D reconstruction. By detecting direct changes in oxygenated muscle tissue, the NIRS vaginal speculum is able to provide insight into how the oxidative capacity of the PFM influences SUI. The small number of units able to provide patient evaluation using these techniques and their cost and relative complexity are major considerations, but if such imaging can optimize diagnosis, treatment allocation, and selection for surgery enhanced imaging techniques may prove to be a worthwhile and cost-effective strategy for assessing and treating SUI.

\section{Introduction}

Advances in the diagnosis and treatment of female stress urinary incontinence (SUI) are being aided by the use of emerging technologies that offer greater understanding of the basic science underlying bladder disease. ${ }^{1}$ This is relevant given that female SUI affects $32-64 \%$ of the female population and that $11.1 \%$ will need to undergo treatment or require surgery in their lifetime. ${ }^{3}$ In addition to providing novel insights on the causal etiology, new technologies offer elements able to enhance diagnosis and tools relevant to optimizing therapy for a large number of women worldwide. ${ }^{4}$

Pregnancy and childbirth are the important causes of pelvic floor injury. Subpopulations of patients are recognized within the spectrum of SUI. SUI can be observed in nulliparous women $4.7 \%$ of the time. ${ }^{4}$ During pregnancy SUI is reported in up to $50 \%$ of women, and additional insults to the pelvic floor are known to occur during labour and delivery. ${ }^{5}$

Epidemiological studies have identified elements related to labour and delivery that influence the frequency of symptoms; however, it is still unclear how much pregnancy contributes to the pathology, whether the predominant effects occur during labour and delivery, and what is the relevance of other risk factors, e.g., maternal age, body weight, genetics, and lifestyle. ${ }^{4}$

Currently, it is believed that partial denervation of the pelvic floor and lineal injury to pelvic floor muscles and connective tissue are caused by mechanical and hormonal changes during pregnancy, as well as physical strain and/or injury during labour and delivery. ${ }^{4}$ Such injuries may cause a woman to develop SUI, anal incontinence, and pelvic organ prolapse (POP). The reported prevalence rate of SUI after cesarean delivery is $6.9 \%$ and after vaginal delivery is $12.2 \%{ }^{4}$

The objective of this review of advanced imaging systems that are currently becoming available is to make clinicians aware of new approaches to diagnosis and treatment that will increase our understanding of the basic science and pathophysiology of SUI in different clinical settings.

\section{Open-configuration magnetic resonance imaging}

The recent development of the open-configuration magnetic resonance imaging (MRO) system allows us to image the pelvic structures in different positions — sitting and upright - that simulate conditions more often linked with urinary leakage. ${ }^{6}$ Specifically, the contributions of gravity in the upright position on organ location, degree of prolapse, and 
the integrity of the pelvic floor musculature can be studied. ${ }^{6}$ Women with SUI suffer from considerable overall laxity of the pelvic floor, which is demonstrated by descent of the bladder neck and rotation of the urethra with straining. Although these findings can be seen in the supine position in some women, recent studies indicate that prolapse is evident more often using MRO images obtained in an upright weight-bearing position, ${ }^{7}$ and women whose supine studies are negative for prolapse have prolapse evident when they are imaged standing. This observation corroborates the clinical observation that women suffering from SUI generally do not complain of leakage in the supine position. ${ }^{6}$

The first described use of magnetic resonance imaging (MRI) was in 1983 in The Lancet; $^{8}$ techniques related to urology have evolved over the last 30 years. In the 1990s, MR techniques were enhanced with faster and vigorous gradients and even higher readout bandwidth. With these progressions, fast imaging sequences were obtained. ${ }^{3}$ DeLancey et al $^{9}$ used MRI to investigate the appearance and shape of the levator ani muscle after vaginal delivery. Sequent MRI was used by Lien et $\mathrm{al}^{10}$ to recognize the levator ani muscle stretch during vaginal birth; it was concluded that the levator ani muscle disorder is related to hard vaginal delivery and older maternal age.

There are many elements of the latest form of imaging, $\mathrm{MRO}$, that will advance our understanding of the basic science of pelvic floor anatomy and function beyond what has been learned to date from conventional fluoroscopic and sonographic methods. ${ }^{11} \mathrm{MRO}$ allows comparatively noninvasive, dynamic assessment of all components of the pelvic floor and the organs in several planes with high softtissue and temporal resolution unaccompanied by the use of ionizing radiation. In addition, MRO readily visualizes the muscular and ligamentous components of the pelvic floor. MRO does have some limitations; these include the current scarcity of MRO units, cost, and the need for radiologists with additional expertise. ${ }^{11}$ Fig. 1 illustrates the ability of MRO to image the pelvic floor in the context of SUI and POP in the supine and standing positions.

\section{D MRI pelvic reconstruction}

$3 \mathrm{D}$ reconstruction can enhance the understanding of anatomical and pathophysiologic alteration in women with pelvic floor disorders. Traditional 2D MRI has been used in many research studies to evaluate the anatomy of the female pelvic floor in cadavers and incontinent women. ${ }^{6,12-16}$ Although not yet comprehensively evaluated, 3D imaging offers an additional basic science parameter through the quantification of muscle volume. It has been suggested that this measure is beneficial in the assessment in healthy women and in those with SUI, prolapse, and other forms of pelvic floor dysfunction. ${ }^{17-19} 3 \mathrm{D}$ imaging provides the most precise representation of the relationships between pelvic floor structures and, hence, is of particular value for surgical planning. ${ }^{19}$ Previous studies have reported 3D reconstruction applied to quantify diminished levator ani muscle mass in patients with pelvic floor dysfunction. ${ }^{20}$ In other areas of urology, image-based $3 \mathrm{D}$ reconstruction has proved helpful as a research technique for centralizing and measuring the volume of tumours in the kidneys and bladder; ; $^{1,21-23}$ however, the use of 3D reconstruction, as with other advanced techniques, requires the use of a defined and reproducible protocol and a method for measuring the 3D renderings. ${ }^{24}$ An example of 3D MRI pelvic reconstruction is shown in Fig. 2. Further protocol and analytic details are provided in the online supplement.

The benefits of 3D reconstruction include the additional information provided for patient-clinician interaction,

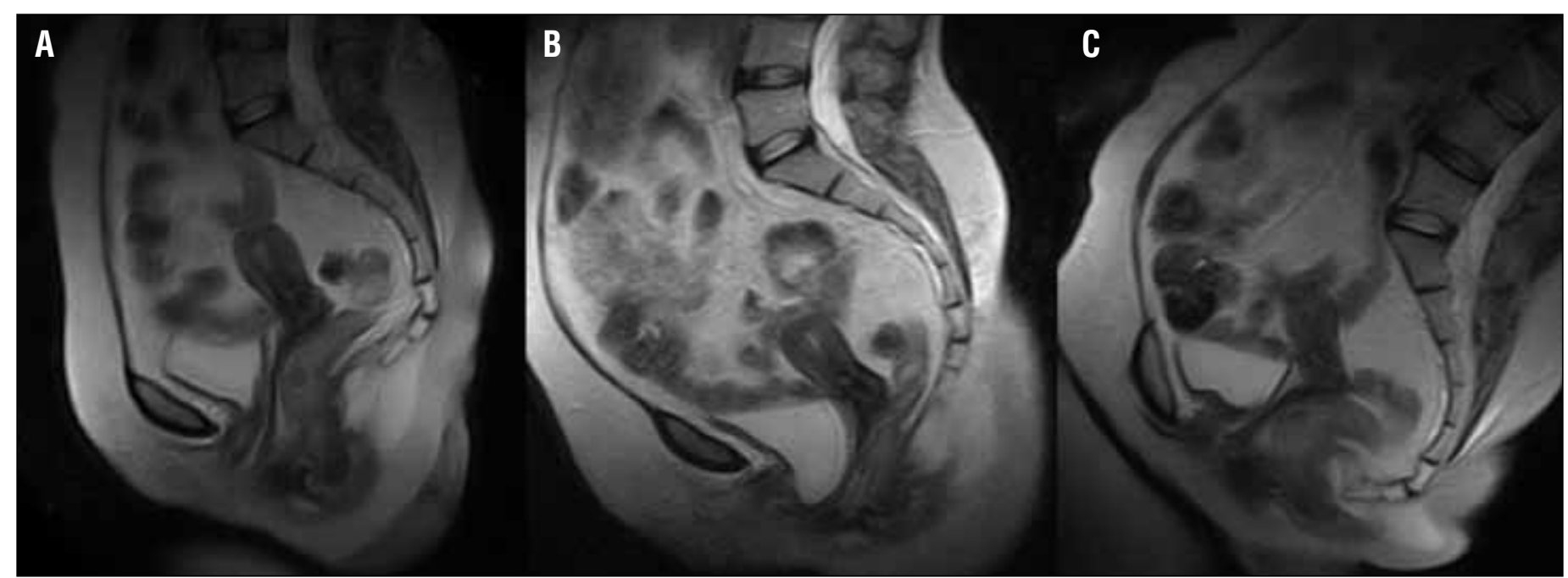

Fig. 1. An example of positional magnetic resonance imaging: $(\boldsymbol{A})$ in the supine position; $(\boldsymbol{B})$ in the standing position; and $(\boldsymbol{C})$ in the sitting position. This provides an example whereby prolapse is evident during imaging by incorporating standing imaging. 


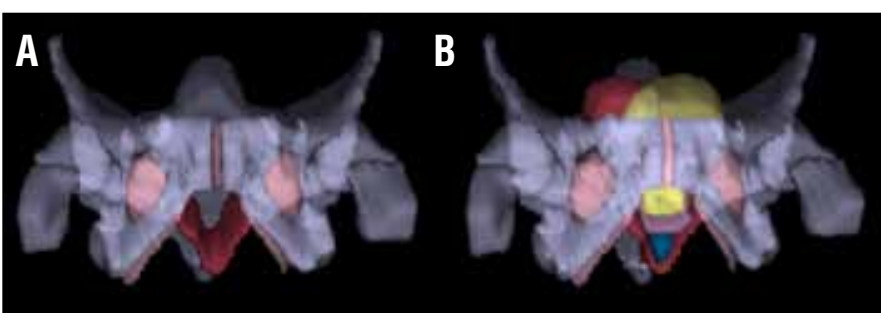

Fig. 2. Three-dimensional imaging: $(\boldsymbol{A})$ reconstruction of pelvic floor muscles; and $(\boldsymbol{B})$ reconstruction of the whole female pelvis.

enhanced surgical planning, medical research, and education. The limitations include cost and complexity of acquiring the initial images and the need for special equipment, including computer hardware and software. ${ }^{25}$ As with other technologies, advances in computer hardware and software will make 3D imaging faster and more cost-effective in the future. When such imaging becomes available it will extend our basic science knowledge related to muscle morphology, bulk, and signal intensity and likely improve allocation of appropriate treatment. ${ }^{19}$

The role of MRI in the assessment of patients with urinary incontinence is anticipated to grow in importance in the future. ${ }^{26}$ The ability of MRI to allow study of the entire muscle in both two and three dimensions is a clear advantage for scientific research. ${ }^{18,19,27}$

\section{Near infrared spectroscopy vaginal speculum}

Near infrared spectroscopy (NIRS) is a validated tool commonly used in research to study the pathophysiology of skeletal muscle in states of health and disease. ${ }^{28}$ However, the use of this technology has only recently entered the field of urology to examine the detrusor and the pelvic floor muscles. ${ }^{29} \mathrm{NIR}$ light is emitted at different wavelengths in the infrared region of 700-1000 nm. At these wavelengths NIRS is able to penetrate biological tissue and immediately detect changes of oxygen concentrations in the hemoglobin chromophores directly within muscle tissue. This allows for sensitive interpretation of targeted muscle oxygen delivery and consumption patterns, providing insight on the functional quality of the targeted muscle. ${ }^{29}$ Accurate assessment of PFM is critical for implementing appropriate management of PFM dysfunction. ${ }^{30}$ By adapting NIRS for use in a vaginal speculum, the hemodynamics of the PFM can be examined in real time, allowing for better understanding of how the physiology of the PFM influences SUI and how to better tailor conservative therapy for SUI management. Further protocol and analytic details are provided in the online supplement.

\section{Conclusion}

The immediate NIRS tracings produced in response to PFM function could be beneficial for biofeedback in conserva- tive management for pelvic floor dysfunction, including POP and SUI. New advances in imaging techniques have the potential to provide objective confirmation of findings assessed by examination, such as levator ani injuries, or structural findings not clear to the clinician, depending on pelvic anatomy, during a clinical examination. They open the door to greater insights into the pathophysiology of SUI through the different techniques described. In addition, as they enter the mainstream they will enable clinicians and researchers to improve on current diagnostic and treatment modalities and also provide repositories of data that can be used to further refine approaches that advance basic science knowledge and enhance our understanding of SUI.

Competing interests: The authors report no competing personal or financial interests.

This paper has been peer reviewed.

\section{References}

1. Stothers L, Macnab AJ. Integration of spectroscopy and imaging to optimize evaluation of voiding dysfunction. Biomed Spectrosc Imaging 2016;5:283-94. https://doi.org/10.3233/BSI-160149

2. Morkved $S$, Bo K. Effect of pelvic floor muscle training during pregnancy and after childbirth on prevention and treatment of urinary incontinence: A systematic review. Br I Sports Med 2014;48:299-310. https://doi.org/10.1136/bisports-2012-091758

3. Kline-Fath B. Prenatal imaging. Newborn Infant Nurs Rev 2007;7:197-204. https://doi.org/10.1053/j. nainr.2007.09.002

4. Groutz A, Helpman L, Gold R, et al. First vaginal delivery at an older age: Does it carry an extra risk for the development of stress urinary incontinence? Neurourol Urodyn 2007;26:779-82. https://doi.org/10.1002/nau.20414

5. Swift SE. The distribution of pelvic organ support in a population of female subjects seen for routine gynecological healthcare. Am J Obstet Gynecol 2000;183:277-85. https://doi.org/10.1067/ mob.2000.107583

6. Fielding JR, Griffiths DJ, Versi E, et al. MR imaging of pelvic floor continence mechanisms in the supine and sitting positions. AJR Am J Roentgenol 1998;171:1607-10. https://doi.org/10.2214/ air. 171.6 .9843296

7. Friedman B, Stothers L, Lazare D. Positional pelvic organ prolapse (POP) evaluation using open, weight-bearing magnetic resonance imaging (MRI). Can Urol Assoc J 2015;9:197-200. https://doi.org/10.5489/cuai.2767

8. Smith FW, Adam AH, Phillips WD. NMR imaging in pregnancy. Lancet 1983;321:61-2. https://doi.org/10.1016/S0140-6736(83)91588-X

9. DeLancey JO, Kearnery $R$, Chou $Q$, et al. The appearance of levator ani muscle abnormalities in magnetic resonance images after vaginal delivery. Obstet Gynecol 2003;101:46-53. https://doi.org/10.1097/00006250-200301000-00012

10. Lien KC, Mooney, B, DeLancey JO, et al. Levator ani muscle stretch induced by simulated vaginal birth. Obstet Gynecol 2004;103:31-40. https://doi.org/10.1097/01.A0G.0000109207.22354.65

11. Woodfield CA, Krishnamoorthy S, Hampton BS, et al. Imaging pelvic floor disorders: Trend toward comprehensive MRI. AJR Am J Roentgenol 2010;194:1640-9. https://doi.org/10.2214/AJR.09.3670

12. Strohbehn $\mathrm{K}$, Ellis JH, Strohbehn JA, et al. Magnetic resonance imaging of the levator ani with anatomic correlation. Obstet Gynecol 1996;87:277-85. https://doi.org/10.1016/0029-7844(95)00410-6

13. Frohlich $B$, Hotzinger $H$, Fritsch $H$. Tomographical anatomy of the pelvis, pelvic floor, and related structures. Clin Anat 1997;104:223-30. https://doi.org/10.1002/(SICI) 1098-2353(1997) 10:4<223::AIDCAl>3.0.C0;2-T

14. Healy JC, Halligan S, Reznek RH, et al. Patterns of prolapse in women with symptoms of pelvic floor weakness: Assessment with MR imaging. Radiology 1997;203:77-81. https://doi.org/10.1148/ radiology.203.1.9122419

15. Lienemann A, Anthuber A, Baron A, et al. Dynamic MR colpocystorectography assessing pelvic-floor descent. Eur Radiol 1997;7:1309-17. https://doi.org/10.1007/s003300050294 
Abdulaziz et al.

16. Fielding JR, Versi E, Mulkern RV, et al. MR imaging of the female pelvic floor in the supine and upright positions. J Magn Reson Imaging 1996;6:961-3. https://doi.org/10.1002/imri.1880060622

17. Hoyte L, Thomas J, Foster RT, et al. Racial differences in pelvic morphology among asymptomatic nulliparous women as seen on three-dimensional magnetic resonance images. Am J Obstet Gynecol 2005;193:2035-40. htrps://doi.org/10.1016/i.ajog.2005.06.060

18. Hoyte L. Schierlitz L, Zou K, et al. Two- and 3-dimensional MRI comparison of levator ani structure, volume, and integrity in women with stress incontinence and prolapse. Am J Obstet Gynecol 2001;185:11-9. https://doi.org/10.1067/mob.2001.116365

19. Fielding JR, Dumanli H, Schreyer AG, et al. MR-based three-dimensional modeling of the normal pelvic floor in women: Quantification of muscle mass. AJR Am J Roentgenol 2000;174:657-60. https://doi.org/10.2214/air.174.3.1740657

20. Chen L, Hsu Y, Ashton-Miller JA, et al. Measurement of the pubic portion of the levator ani muscle in women with unilateral defects in 3-D models from MR images. Int J Gynaecol Obstet 2006;92:234-41. https://doi.org/10.1016/i.i.jgo.2005.12.001

21. Kikinis R, Gleason PL, Moriarty TM, et al. Computer-assisted interactive three-dimensional planning for neurosurgical procedures. Neurosurgery 1996;38:640-9. https://doi.org/10.1227/00006123 199604000-00003

22. Schreyer AG, Fielding JR, Warfield SK, et al. Virtual CT cystoscopy: Color mapping of bladder wall thickness. Invest Radiol 2000;35:331-4. https://doi.org/10.1097/00004424-200005000-00008

23. Stenzl A, Frank R, Eder R, et al. 3-dimensional computerized tomography and virtual reality endoscopy of the reconstructed lower urinary tract. J Urol 1998;1593:741-6. https://doi.org/10.1016/S0022$5347(01) 63716-9$
24. Cline HE, Lorensen WE, Ludke $S$, et al. Two algorithms for the three-dimensional reconstruction of tomograms. Med Phys 1988;15:320-7. https://doi.org/10.1118/1.596225

25. Rengier F, Mehndiratta A, von Tenqq-Kobligk H, et al. 3D printing based on imaging data: Review of medical applications. Int J Comput Assist Radiol Surg 2010;5:335-41. https://doi.org/10.1007/ s1 1548-010-0476-x

26. Macura KJ, Genadry RR, Bluemke DA. MR imaging of the female urethra and supporting ligaments in assessment of urinary incontinence: Spectrum of abnormalities. Radiographics 2006;26:1135-49. https://doi.org/10.1148/rg.264055133

27. Tunn R, DeLancey JO, Howard $D$, et al. MR imaging of levator ani muscle recovery following vaginal delivery. Int Urogynecol J Pelvic Floor Dysfunct 1999; 10:300-7. https://doi.org/10.1007/s001929970006

28. Grassi B, Quaresima V. Near-infrared spectroscopy and skeletal muscle oxidative function in vivo in health and disease: A review from an exercise physiology perspective. J Biomed Opt 2016;21:091313-091313. https://doi.org/10.1117/1.JB0.21.9.091313

29. Macnab AJ. The evolution of near infrared spectroscopy. Biomed Spectrosc Imaging 2014;3:311-44.

30. Bø K, Frawley HC, Haylen BT, et al. An International Urogynecological Association (IUGA)/ International Continence Society (ICS) joint report on the terminology for the conservative and nonpharmacological management of female pelvic floor dysfunction. Int Urogynecol J 2017;28:191-213. hitps://doi.org/10.1007/s00192-016-3123-4

Correspondence: Dr. Lynn Stothers, Department of Urologic Sciences, University of British Columbia, Vancouver, BC, Canada; lynns@mail.ubc.ca 